\title{
High-Throughput Sequencing Reveals miRNAs Affecting Follicle Development in Chicken
}

\author{
Quan Zhang*, Shanshan Li, Bingwang Du \\ Department of Animal Breeding and Genetics, Agricultural College, Guangdong Ocean University, Zhanjiang, P. R. China
}

Email address:

gdouzq@foxmail.com (Quan Zhang)

${ }^{*}$ Corresponding author

To cite this article:

Quan Zhang, Shanshan Li, Bingwang Du. High-Throughput Sequencing Reveals miRNAs Affecting Follicle Development in Chicken. International Journal of Genetics and Genomics. Vol. 5, No. 6, 2017, pp. 76-84. doi: 10.11648/j.ijgg.20170506.12

Received: October 5, 2017; Accepted: October 19, 2017; Published: November 16, 2017

\begin{abstract}
As the derivative of chicken skin, hair follicle is capable of self-renew. Its proliferation and differentiation result in hair formation. MicroRNAs (miRNAs) can effectively regulate gene expression at the post-transcriptional level and play a critical role in tissue growth, development. In this study, we used next generation sequencing technology sequenced miRNAs of the hair follicle derived from the 13 day-old chicken (Gallus gallus) embryos in which from Kirin chicken and Huaixiang chicken that feathers having morphogenesis with significantly different curling. A population of conserved miRNAs was identified. These conserved miRNAs were derived from 638 homologous hairpin precursors across 5 animal species. We identified a total of 645 miRNAs in the chicken embryos. Among them, 11 differentially expressed miRNAs were identified $(> \pm 2$ Fold, $p$ value $<0.05$ ) by comparing Kirin chicken and Huaixiang chicken. Several gene ontology (GO) biology processes and the WNT, BMP and TGF- $\beta$ signaling pathways were found to be differentially expressed miRNAs as part of hair follicle development process. The miR-1623 has an effect on WNT4 and involved in hair follicle cell development. This study has identified miRNAs that associated with the chick embryonic hair follicle development and identified some target miRNAs for further research into their role played in feather growth.
\end{abstract}

Keywords: Chicken Embryo, Follicle, Mirnas

\section{Introduction}

MicroRNAs (miRNAs) are small non-coding RNA molecules that suppress gene expression post-transcriptionally, and function important roles in diverse biological processes [1]. Hundreds of miRNA genes have been found in diverse animals, and many of these are phylogenetically conserved [2]. In addition to endogenous presence in cells, miRNAs can also be actively released into extracellular fluids through exosomes or microvesicles [3, 4]. Consequently, miRNA research has become a hot spot in the field of biological for explaining molecular formation mechanisms [5] and important traits of animals [6-9]. The skin plays an important protection role in animal existence and it evolves with the animal bifurcation.

The feather is one of the most complex integumentary appendages due to the extensive diversity in shape, size, arrangement and pigmentation, and is therefore an excellent model for evolutionary and developmental biology as variations can occur at each step of development and differentiation [10-13]. The Kirin chicken can adapt to high-temperature environment because of unique frizzled feather branching structure characteristic, rachis stout and outwardly curved, barbs short sparse, feather hook can't connect with the back edge of the adjacent twig lead to pinna can not closed. Recent advances that frizzled feather is caused by KRT75 mutation reside in autosomal, belong to incomplete dominant inheritance [14]. Feathers develop from the hair follicle, therefore the hair follicles numbers, diameter with feathers growth have a direct relationship [15, 16]. And miRNAs connected with hair follicles developmental processes [5, 17] and regulate hair follicle development and hair growth [18, 19]

In this study, we investigated the expression profile of miRNAs in the follicles of 13-day chicken embryos from the Kirin chicken (KRC) and Huaixiang chicken (HXC). The results demonstrate that chicken embryonic follicle contains large amounts of miRNAs. 


\section{Materials and Methods}

\subsection{Ethics Statement}

All chicken embryos experiments were approved and reviewed by the local ethical committee and the procedures in this study followed the guidelines of the Guangdong Ocean University Animal Care and Use Committee. To minimize the suffering of animals, sodium pentobarbital anesthesia was used before the collection of chicken skin hair follicles samples.

\subsection{Collection of Chicken Embryonic Follicle Samples}

Fertile eggs were collected from 45-wk-old KRC and $\mathrm{HXC}$. Fertile eggs were incubated at $37.8^{\circ} \mathrm{C}$ with 65 to $75 \%$ humidity and intermittent rotations, which to provided $2-3 \mathrm{~cm}$ skin tissue were obtained from the back of the body of 13-day chicken embryos, separately. Samples of hair follicle stored at $-80^{\circ} \mathrm{C}$ until used.

\subsection{Small RNA Library Preparation and Sequencing}

Collected feather follicle from six chicken embryos at the age of 13-day were used to construct two small RNA libraries in this study. These samples included three KRC ones and three HXC, respectively.

Total RNA was extracted from the follicle using TruSeq Small RNA Sample Pre Kits (Illumine, San Diego, USA) according to the manufacturer's instructions. Total RNA quality was checked with a Bioanalyzer 2100 (Agilent Technologies, USA). The RIN was $>8.0$ and A260/A280 was $>$ 2.1 for all samples. The equal concentration total RNAs of six samples were constructed small RNA libraries by TruSeq Small RNA Sample prep Kit of illumina. The overall flow of the sequencing procedure is as follows: small RNAs ranging from 18 to $35 \mathrm{nt}$ in length was purified from $15 \%$ polyacrylamide gels, then ligated to '5' and 3' adapters. Reverse transcription was performed, and followed by PCR amplification. The purified PCR products ( $140 \mathrm{bp})$ were used directly for cluster generation and sequencing analysis using the Illumina's 2000 Sequencer according to the manufacturer's instructions (Personalbio, ShangHai, China).

\subsection{Sequence Data Analysis}

Sequence data analysis was done using AGGT101-miR tool. After deleting poor quality reads, adaptor pollution reads and reads less than $18 \mathrm{nt}$, the clean reads were obtained.

The clean reads of small RNAs were aligned to the reference chicken (G. gallus) genome to identify known miRNAs. The sequences that matched perfectly to known miRNAs (miRBase V21.0) were determined as conserved miRNAs. Other small RNAs (rRNA, tRNA, snRNA and snoRNA) were annotated by blasting against the Rfram, Repbase and ncRNA databases.

The unannotated small RNA sequences were aligned to the reference chicken (G. gallus) genome to find potential precursor sequences for novel miRNAs. Novel miRNAs were predicted by RNA-fold tools following the criteria: (1) number of nucleotides in one bulge in stem $(<=12)$; (2) number of base pairs in the stem region of the predicted hairpin $(>=16)$; (3) cutoff of free energy $(\mathrm{kcal} / \mathrm{mol}<=-15)$; (4) length of hairpin (up and down stems + terminal loop $>=50$ ); (5) length of hairpin loop $(<=20)$; (6) number of nucleotides in one bulge in mature region $(<=8)$; (7) number of biased errors in one bulge in mature region $(<=4)$; $(8)$ number of biased bulges in mature region $(<=2)$; (9) number of errors in mature region $(<=7)$; (10) number of base pairs in the mature region of the predicted hairpin ( $>=12)$; (11)percent of mature in stem $(>=80)$. Furthermore, the raw reads $>=10$ at least.

To identify differentially expressed miRNAs, the number of conserved miRNAs was normalized to the total number of reads in each sample that matched the chicken (Gallus Gallus) genome. P-values for differentially expressed miRNAs (KRC/HXC) were calculated by Fisher's exact-test and Chi square $(2 \times 2)$ test.

\subsection{Mirna Target Prediction and Functional Analysis}

Target genes of differentially expressed miRNAs were predicted by Target Scan and miRanada. To acquire higher prediction accuracy, only common target genes were considered. Gene Ontology (GO) annotation and Kyoto Encyclopedia of Genes and Genomes (KEGG) pathway analysis were retrieved using DAVID (http://david.abcc.ncifcrf.gov/).

\subsection{Quantitative RT-PCR}

Total RNAs of sampled follicle were reverse-transcribed by PrimeScript ${ }^{\circledR}$ RT reagent Kit (TAKARA, DRR037A). The primers (Table 1) were designed by Primer 5.0 (ABI). 5ul RT reaction system included: denatured RNA and RT primer (2 uM) 3.0ul, $5 \times$ PrimeScript ${ }^{\circledR B u f f e r} 1.0 u l$, RNase Free $\mathrm{dH}_{2} \mathrm{O}$ 0.6ul, PrimeScript ${ }^{\circledR}$ RT Enzyme Mix I 0.4ul. The RT reactions were performed as follows: $42^{\circ} \mathrm{C}$ for 15 minutes, $85^{\circ} \mathrm{C}$ for 5 seconds and hold at $4^{\circ} \mathrm{C}$. $20 \mathrm{ul}$ real-time PCR reaction system included: $2 \times$ SYBR Green Mix with $\mathrm{ROX}$ 10.0ul, dd $\mathrm{d}_{2} \mathrm{O} 8.2 \mathrm{ul}$, Primer mix (10 uM) 0.8ul, cDNA $1 \mathrm{ul}$. The PCR reactions were performed as follows: $50^{\circ} \mathrm{C}$ for 2 minutes, $95^{\circ} \mathrm{C}$ for 2 minutes, then 40 cycles with $94^{\circ} \mathrm{C}$ for 15 seconds and $60^{\circ} \mathrm{C}$ for 30 seconds.

Table 1. The list of RT-qPCR primer.

\begin{tabular}{lll}
\hline Primer & sequences (5'to3') & bp \\
\hline miR-1623 & ACCGCAGGCACAGACAGGCAGT & 22 \\
miR-U6 & TGCTTTGGCAGCACATATACCAA & 23 \\
Reverse Primer & GATCGCCCTTCTACGTCGTAT & 21 \\
WNT4-F & TCTACGCCATCTCTTCAGCA & 20 \\
WNT4-R & AGGCAATGTTATCGGAGCAG & 20 \\
$\beta$-actin-F & TGCCAGGGTACATTGTGGTA & 20 \\
$\beta$-actin-R & TGCGTGACATCAAGGAGAAG & 20 \\
\hline
\end{tabular}

All experiments were performed on ABI 7900 HT sequence detection system. Each reaction was carried out with 3 replicates. snRNA U6 was used as the control for RT-qPCR. The relative expression level of each miRNA to U6 snRNA was normalized as $\Delta \mathrm{Cp}=\mathrm{Cq}$ miRNA - Cq U6RNA [20]. Comparison of relative expression level in different stages 
was determined using the $2-^{\Delta \Delta \mathrm{Cp}}$ method [21]. Statistical significance analysis of the expression change was performed by one-way ANOVA in SPSS 20.0.

\section{Results}

\subsection{Small RNA Library Construction and Sequencing}

To investigate the miRNA expression profile in chicken follicle, High-throughput HiSeq 2000 sequencing yielded 9,714,611 (HXC) and 10,875,477 (KRC) raw reads on average for each group small RNA libraries. After filtered low quality sequences, 9,134,499 (HXC) and 7,919,788 (KRC) clean reads for each group were obtained respectively (Table 2). The histograms of the reads length distribution showed majority were $20 \mathrm{nt} \sim 24 \mathrm{nt}$ (Figure 1). Of these, 35,705 (HXC) and 52,201 (KRC) unique small RNAs were identified.

Table 2. The hair follicles small RNA sequencing reads of Kirin chicken and Huaixiang chicken.

\begin{tabular}{|c|c|c|c|c|c|c|}
\hline Sample & HXC1 & HXC2 & HXC3 & KRC1 & KRC2 & KRC3 \\
\hline raw reads & $9,873,177$ & $9,556,045$ & $11,410,76$ & $11,278,066$ & $11,337,531$ & $10,010,835$ \\
\hline clean reads & $9,318,257$ & $8,950,741$ & $10,596,63$ & $8,282,543$ & $8,141,768$ & $7,335,054$ \\
\hline $15-30 \mathrm{nt}$ reads & $8,881,208$ & $7,869,359$ & $9,238,098$ & $7,733,489$ & $7,519,340$ & $6,329,447$ \\
\hline unique reads & 36,938 & 31,801 & 38,376 & 56,408 & 53,568 & 46,628 \\
\hline miRNA & 540 & 525 & 572 & 524 & 543 & 520 \\
\hline
\end{tabular}

HXC: wild feather of Huaixiang chickens; KRC: frizzle feather of Kirin chickens.

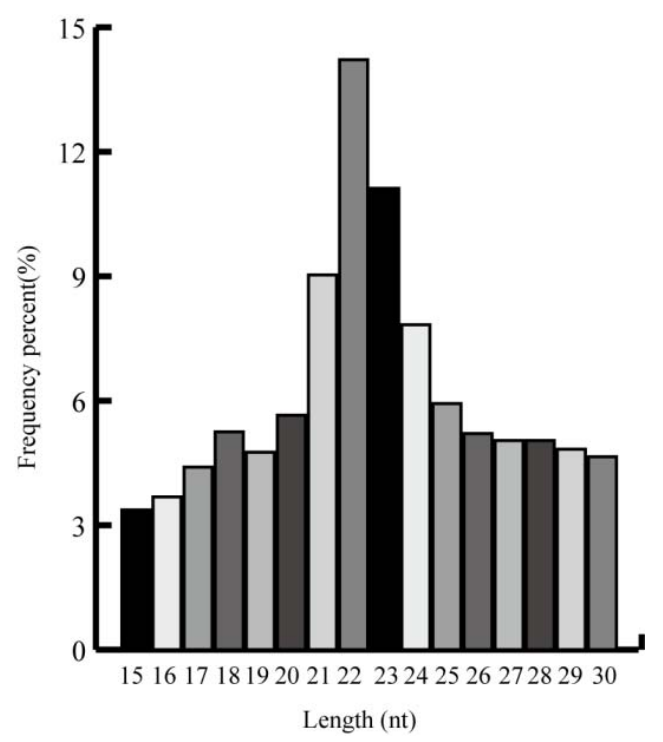

Figure 1. The histograms of the RNA-seq reads length distribution from $15 \mathrm{nt}$ to $30 \mathrm{nt}$.

\subsection{Identification of Conserved Mirnas}

To identify conserved miRNAs in chicken follicle, the small RNAs were aligned to current miRBase (Release V21.0). Sequences with perfect matching to known chicken (Gallus Gallus) miRNAs were considered as conserved miRNAs. In total, 645 conserved sequences were annotated as chicken miRNAs. To obtain higher reliable results, only the miRNAs with raw reads $>10$ at least were considered. Then 290 were sorted as common miRNAs, with only one KRC-specific miRNA (gga-miR-1682). All 291 conserved miRNAs detected by sequencing were listed in Table 3.

Table 3 The conserved miRNAs expressed in the chicken follicle

\begin{tabular}{ll}
\hline miRNA_name & miRNA_seq \\
\hline gga-miR-6586-5p & TGCTGCCAGATAGAAGTTCACCT \\
gga-miR-1467-5p & TCTCAGCTACATCGGTGTAAATC \\
gga-miR-101-1-5p & CGGTTATCATGGTACCGGTGCTGT \\
gga-miR-1329-3p & CCTCGTAGCTTGATCACGATAT \\
\hline
\end{tabular}

Continuous the above Table 3

gga-miR-3607-5p gga-miR-6544-5p gga-miR-6555-5p gga-miR-6694-3p gga-miR-1800 gga-miR-1737 gga-miR-1559-3p gga-miR-429-5p gga-miR-6550-3p gga-miR-19b-5p gga-miR-6548-5p gga-miR-29a-3p gga-miR-6590-3p gga-miR-23b-5p gga-miR-2128 gga-miR-6575-5p gga-miR-1727 gga-miR-1591-5p gga-miR-128-2-5p gga-miR-1781-3p gga-miR-6548-3p gga-miR-6516-3p gga-miR-1626-3p gga-miR-30b-3p gga-miR-1666 gga-miR-1644 gga-miR-135a-3-3p gga-miR-7460-3p gga-miR-1698 gga-miR-6582-3p gga-miR-1808 gga-miR-6566-5p gga-miR-6565-3p gga-miR-33-3p gga-miR-6631-5p gga-miR-1649-5p gga-miR-6648-3p gga-miR-16-2-3p gga-miR-6669-3p gga-miR-1684a-3p gga-miR-3532-3p gga-miR-1651-3p gga-miR-1805-3p gga-miR-1707 gga-miR-1769-3p
TACATATGATGAGCTTTGCAGT

TTCAGAAAAGGATATGAATTGT GATCTGCAGAGCCCACAACTAG TTAAGAGTAGGGATTCTGTTCC ACTACGTGATGCGATCTGATG CAGCACTGCTGCGCTCGGTG AGTTACATGTATGCATCGAGCA GTCTTACCAGGCAAAGTTAGA GCTCCACCCTCGGCTGCTTTGA AGTTTTGCAGGTTTGCATCCCAGC AACAACAGCTGCGTGCCATGCC TAGCACCATTTGAAATCGGTTA TTACTTCTGTTCCTGATCATCA TGGGTTCCTGGCATGATGATTT CAGTGACGTCTCTTCCCCGCAGT TTGTCAGCTTGGGGAAGCTCTT AAGCTGCTCTAATGAACTGAAG TGATTCATTGCCTGGCTCTGCA GGGGGCCGTTACACTGTAAGAGA TTTAAATCATGCAGCTGTTGA CAGAGGTGCCCCGCTGTTGCTGT TGGTCATGTATGATACTGCACA TCTGGAAGTTGCCCTGGACGTGT CTGGGGGGTGGATGTTTACTTC TAACGCCACGGGGCTGAGGCTG TCTGTTGTGCAGGGCTGTGCTCT ATGTAGGGCGAAAAGCCATGGG CCTGACTGAGCTCTGCTTTCTC CGAGGCTGCGGCAATCCCTGCGC CACTCTGGGTGTTTCTCTTGCAG TGTTGGGAATGAATACATATTGT TGAGGCCCGATGTGTCATTCCTGGA TCTGTGCTTGTGTACTCATAGT CAATGTTCCTGCAGTGCAGTA GAAGAGAATGCTGTGGTTCTGC TCCTGCAGAAGGTGCGGCTGTGT TCCGGCATTCTGAACGCTCCT CCCAATATTATTGTGCTGCTT TGCAGCCTGGCCGTATCTCAGT AAGTATGAGGAAATGGAGCTCT TTGGAGGCTGCAGTGTCATGGT TTGCTTTTGTTGGCCTCTGCTGT TGTATTGGAACACTACAGCTCC TTTGAGCGGGATCTGTTATCGTG AGTGTGAAATCTGCCTGAAAGT 


\begin{tabular}{|c|c|}
\hline \multicolumn{2}{|c|}{ Continuous the above Table 3} \\
\hline gga-miR-1558 & CTGCTGTGATGGGAGCTCTGAGCAG \\
\hline gga-miR-365-1-5p & AGGGACTTTTGGGGGCAGATGTG \\
\hline gga-miR-133b & TTTGGTCCCCTTCAACCAGCTA \\
\hline gga-miR-1574-3p & ACAGGAGGATGTCAGGAAGCTTC \\
\hline gga-miR-1623 & ACCGCAGGCACAGACAGGCAGT \\
\hline gga-miR-1663-3p & TGGCATCCAGAACAGCGGTAC \\
\hline gga-miR-460b-5p & TCCTCATTGTACATGCTGTGTG \\
\hline gga-miR-153-3p & TTGCATAGTCACAAAAGTGATCGT \\
\hline gga-miR-1716 & AGCGGGGCGGCTGTGAGCTGAGCT \\
\hline gga-miR-6549-5p & AGCCTTCTGTTGTGCATCTGAGA \\
\hline gga-miR-551-3p & GCGACCCATACTTGGTTTCAGT \\
\hline gga-miR-138-5p & AGCTGGTGTTGTGAATCAGGC \\
\hline gga-miR-6710-3p & AAACTGTTCTCTTCCATCTAGT \\
\hline gga-miR-1458 & TTCCTGTGATGCTCATGAGA \\
\hline gga-miR-1729-3p & CTACTCGGTGAGTAAGGATAGC \\
\hline gga-miR-1663-5p & TACCGCTGTCCTGGGTGCCTGG \\
\hline gga-miR-34c-5p & AGGCAGTGTAGTTAGCTGATTGT \\
\hline gga-miR-6543-5p & GCTGTTACCTGAGAGAAGACGCTG \\
\hline gga-let-7g-3p & CTGTACAGGCCACTGCCTTGC \\
\hline gga-miR-3594-3p & TCTGCATCGCTGGGCTGTGTCCT \\
\hline gga-miR-135a-2-3p & TGTAGGGATGGAAGCCATGA \\
\hline gga-miR-9-3p & TAAAGCTAGAGAACCGAATGTA \\
\hline gga-miR-196-1-3p & ACAAGAACATCAAACTACCTGA \\
\hline gga-miR-6670-5p & TGGAATGATGATCTATATTCTGAGT \\
\hline gga-miR-138-1-3p & GCTACTTCACAACACCAGGGT \\
\hline gga-miR-130c-5p & CCTTTTTATGTTGTACTACTAG \\
\hline gga-miR-1747-5p & TGCACTTGAATGGAGTTCTGGGT \\
\hline gga-miR-1563 & GCACATGATGAGGAAGCACTGAAACTGAC \\
\hline gga-miR-6660-3p & CTGACATGGCTCTGCTCCGCAGT \\
\hline gga-miR-1569 & TGTTTGGGACGTTGCTCTGCAG \\
\hline gga-miR-21-3p & AACAACAGTCGGTAGGCTGTCT \\
\hline gga-miR-6615-3p & TGGCACTGATGTGTTCTCCACA \\
\hline gga-miR-1703-5p & AGAGGCTGTAGGTCCCGTGCTTT \\
\hline gga-miR-137-3p & TTATTGCTGGAGAATACGCGTAG \\
\hline gga-miR-1635 & TGCCCAGGCTGTGCTGTGCTCTGG \\
\hline gga-miR-460a-3p & CACAGCGCATACAATGTGGATT \\
\hline gga-miR-181b-2-3p & CTCACTGATCAATGAATGCAAA \\
\hline gga-miR-1724 & TGCTGAGCGTTGGCTGCGCTGC \\
\hline gga-miR-15c-3p & CAGACCATTCTGGGCTGCCTCA \\
\hline gga-miR-1662 & TTGACATCATCATACTTGGGAT \\
\hline gga-miR-6604-5p & TGGCACGGTGCTAGGGATTTCTGT \\
\hline gga-miR-1731-5p & ACTTGACTGCAGGCACTGCTGCT \\
\hline gga-miR-146b-3p & TGCCCTATGGATTCAGTTCTGC \\
\hline gga-miR-1451-3p & CGTAACTCGCTGCTGTGAGAGGT \\
\hline gga-miR-6543-3p & CCTCCTTTCAGGTCACTGTAGG \\
\hline gga-miR-133a-5p & AGCTGGTAAAATGGAACCAAAT \\
\hline gga-miR-138-2-3p & GCTATTTCACTACACCAGGGT \\
\hline gga-miR-1551-5p & CTAGCAGCAAAAAGAACTTCAGA \\
\hline gga-miR-490-3p & CAACCTGGAGGACTCCATGCTG \\
\hline gga-miR-489-3p & TGACATCATATGTACGGCTGCT \\
\hline gga-let-7f-3p & СТATACAATCTATTGCCTTCCT \\
\hline gga-miR-3538 & GTTCGGTGATGAAACCATGGAATA \\
\hline gga-miR-147 & GTGTGCGGAAATGCTTCTGCTA \\
\hline gga-miR-194 & TGTAACAGCAACTCCATGTGGAC \\
\hline gga-miR-24-5p & GTGCCTACTGAGCTGATATCAGT \\
\hline gga-let-7j-3p & CTATACAGTCTATTGCCTTCCT \\
\hline gga-miR-6557-3p & CGCGCCGATTGTCTCCTCCGGGCA \\
\hline gga-miR-16-1-3p & CCAGTATTAACTGTGCTGCTGAA \\
\hline gga-let-7c-3p & CTGTACAACCTTCTAGCTTTCC \\
\hline gga-miR-3529 & AGGCAGACTGTGACTTGTTGT \\
\hline gga-miR-99a-3p & CAAGCTCGCTTCTATGGGTCTGT \\
\hline gga-miR-15b-3p & CGAATCATTATTTGCTGCTTTA \\
\hline gga-miR-3607-3p & ATACTGTAAACGCTTTCTGATG \\
\hline gga-miR-1712-3p & TTCAGTTATCAGTGGAGTTTGG \\
\hline gga-miR-199b & CAGTAGTCTGCACATT \\
\hline gga-let-7a-2-3p & CTGTACAACCTCCTAGCTTTCC \\
\hline gga-miR-6615-5p & TTGGGGACACCATCAGAACAGCCACA \\
\hline
\end{tabular}

\begin{tabular}{|c|c|}
\hline \multicolumn{2}{|c|}{ Continuous the above Table 3} \\
\hline gga-miR-92-5p & AGGTTGGGATCAGTTGCAATGCT \\
\hline gga-miR-22-5p & AGTTCTTCAGTGGCAAGCTTT \\
\hline gga-miR-301b-3p & CAGTGCAATAGTATTGTCAAAGCATT \\
\hline gga-miR-6599-3p & TGACGGATCCTGGCTCCCTCCG \\
\hline gga-miR-33-5p & GTGCATTGTAGTTGCATTGC \\
\hline gga-miR-193a-3p & AACTGGCCTACAAAGTCCCAGT \\
\hline gga-miR-1786 & ATTCTTTTCTGCTGTGTTACT \\
\hline gga-miR-20b-3p & ACTGTAATGTGGGCACTTAC \\
\hline gga-miR-142-5p & CATAAAGTAGAAAGCACTACT \\
\hline gga-miR-3523 & CCGCGCAGTGCCTCGTCCTCGA \\
\hline gga-miR-3525 & CAGCCATTCTGCGATTCTGTGA \\
\hline gga-miR-1744-3p & ACTTCAACAGGAGCAAGACTGA \\
\hline gga-miR-181b-1-3p & CTCACTGAACAATGAATGCAA \\
\hline gga-miR-19a-5p & ю·eTAGTTTTGCATAGTTGCACT \\
\hline gga-miR-1684b-3p & AAGTATGAGGAAATGGAGATCT \\
\hline gga-miR-2188-3p & GATATATGTGGTCGGACCTAT \\
\hline gga-miR-142-3p & TGTAGTGTTTCCTACTTTATGG \\
\hline gga-miR-1625-5p & TGGACCAGGGCTCTTCCTGCTGGCT \\
\hline gga-miR-1560-3p & GCATCTCTGGACGCGCTCGTTC \\
\hline gga-miR-1674 & GGGCTATGATGCTGGATTTTCTGAGCA \\
\hline gga-miR-1677-5p & TCCTGCACCGCTGAAGTCAAT \\
\hline gga-miR-17-3p & CTGCAGTGAAGGCACTTGTAGCT \\
\hline gga-miR-130c-3p & CAGTGCAATGTTAAAAGGGCATT \\
\hline gga-miR-26a-3p & CCTATTCTTGGTTACTTGCACT \\
\hline gga-miR-32-3p & CAATTTAGTGTGTGCGATACT \\
\hline gga-miR-1664-3p & TTCTGTGACCTCATTTACCTCC \\
\hline gga-miR-18a-3p & ACTGCCCTAAGTGCTCCTTCTGG \\
\hline gga-miR-15a & TAGCAGCACATAATGGTTTGTG \\
\hline gga-miR-190a-5p & TGATATGTTTGATATATTAGGTTG \\
\hline gga-miR-2954 & CATCCCCATTCCACTCCTAGCAGTT \\
\hline gga-miR-128-1-5p & CGGGGCCGTAACACTGTCTGAGA \\
\hline gga-miR-146a-3p & ACCCATGGGGCTCAGTTCTTCA \\
\hline gga-miR-133c-3p & TTTGGTCCCCTTCAACCAGCTG \\
\hline gga-miR-383-5p & AGATCAGAAGGTGATTGTGGCT \\
\hline gga-miR-130a-5p & GCCCTTTTTCTGTTGTACTACT \\
\hline gga-miR-1779 & AGACGTGGACTGGAACACCTGAG \\
\hline gga-miR-130a-3p & CAGTGCAATATTAAAAGGGCA \\
\hline gga-miR-15b-5p & TAGCAGCACATCATGGTTTGCA \\
\hline gga-miR-27b-5p & AGAGCTTAGCTGATTGGTGAACA \\
\hline gga-miR-375 & TTTGTTCGTTCGGCTCGCGTTA \\
\hline gga-miR-1416-5p & TCCTTAACTCATGCCGCTGTG \\
\hline gga-let-7d & AGAGGTAGTGGGTTGCATAGTT \\
\hline gga-miR-301b-5p & GCTCTGACTTTATTGCACTACT \\
\hline gga-miR-10b-3p & ACAGATTCGATTCTAGGGGAAT \\
\hline gga-miR-301a-3p & CAGTGCAATAATATTGTCAAAGCATT \\
\hline gga-miR-6542-3p & ACGGGACAGTGCTGAAGACT \\
\hline gga-miR-184-3p & TGGACGGAGAACTGATAAGGGT \\
\hline gga-miR-2131-5p & ATGCAGAAGTGCACGGAAACAGCT \\
\hline gga-miR-1306-5p & CCACCTCCCCTGCAAACGTCCA \\
\hline gga-miR-18b-3p & TACTGCCСТАAATGCTCСТTCT \\
\hline gga-miR-1456-5p & AAAGGACGGAGGCGGCCCGCGC \\
\hline gga-miR-135a-5p & TATGGCTTTTTATTCCTATGTG \\
\hline gga-miR-187-3p & TCGTGTCTTGTGTTGCAGCCAGT \\
\hline gga-miR-18b-5p & TAAGGTGCATCTAGTGCAGT \\
\hline gga-miR-34a-5p & TGGCAGTGTCTTAGCTGGTTGT \\
\hline gga-miR-365-2-5p & AGGGACTTTCAGGGGCAGCTGTG \\
\hline gga-miR-200b-5p & CATCTTACTGGGCAGCATTGGA \\
\hline gga-miR-219b & CACAAGAATTGCGTTTGGACAAT \\
\hline gga-miR-193b-3p & AACTGGCCCACAAAGTCCCGCT \\
\hline gga-miR-1306-3p & TGGACGTTGGCTCTGGTGGTGA \\
\hline gga-miR-2131-3p & CTGTTACTGTTCTTCTGATGG \\
\hline gga-miR-458a-3p & ATAGCTCTTTGAATGGTACTGC \\
\hline gga-miR-122-5p & TGGAGTGTGACAATGGTGTTTG \\
\hline gga-miR-223 & TGTCAGTTTGTCAAATACCCCA \\
\hline gga-miR-144-5p & GGATATCATCATATACTGTAAGT \\
\hline gga-miR-1451-5p & TCGCACAGGAGCAAGTTACCGC \\
\hline gga-miR-301a-5p & GCTCTGACAATGTTGCACTACT \\
\hline
\end{tabular}




\begin{tabular}{|c|c|}
\hline \multicolumn{2}{|c|}{ Continuous the above Table 3} \\
\hline gga-miR-215-5p & ATGACCTATGAATTGACAGACT \\
\hline gga-miR-133a-3p & TTTGGTCCCCTTCAACCAGCTGT \\
\hline gga-miR-1329-5p & TACAGTGATCACGTTACGATGGAT \\
\hline gga-miR-30c-2-3p & CTGGGAGAAGGCTGTTTACTCT \\
\hline gga-miR-148a-5p & AAAGTTCTGTGACACTCAGACT \\
\hline gga-miR-1711 & GGTGCAGTGCTGCATCTCTGG \\
\hline gga-miR-183 & TATGGCACTGGTAGAATTCACT \\
\hline gga-miR-1729-5p & ATCCCTTACTCACATGAGTAGT \\
\hline gga-let-7k-3p & СТАТАCAАТСТАСТGTCTТTCT \\
\hline gga-miR-32-5p & TATTGCACATTACTAAGTTGCA \\
\hline gga-miR-206 & TGGAATGTAAGGAAGTGTGTGG \\
\hline gga-miR-10a-3p & CAAATTCGTATCTAGGGGAAT \\
\hline gga-miR-1b-3p & TGGAATGTTAAGAAGTATGTAT \\
\hline gga-miR-30a-3p & CTTTCAGTCGGATGTTTGCAGC \\
\hline gga-miR-499-5p & TTAAGACTTGTAGTGATGTTTA \\
\hline gga-miR-221-5p & ACCTGGCATACAATGTAGATTTCTGT \\
\hline gga-miR-455-3p & GCAGTCCATGGGCATATACACC \\
\hline gga-miR-19a-3p & TGTGCAAATCTATGCAAAACTGT \\
\hline gga-miR-193a-5p & TGGGTCTTTGCGGGCGAGATGA \\
\hline gga-miR-1552-3p & CTAGCTGCTCTGCACTGACTGT \\
\hline gga-miR-6606-5p & GAGGAGCGGGAGGAGCGGGA \\
\hline gga-miR-181a-3p & ACCATCGACCGTTGATTGTACC \\
\hline gga-miR-15c-5p & TAGCAGCACATCATGGTTTGTA \\
\hline gga-miR-30c-1-3p & TGGGAGAGGATTGTTTACGCCT \\
\hline gga-miR-18a-5p & TAAGGTGCATCTAGTGCAGATAG \\
\hline gga-miR-31-5p & AGGCAAGATGTTGGCATAGCTG \\
\hline gga-miR-1552-5p & TTAGTGCGCGGTAAGCTAGGGTG \\
\hline gga-miR-146c-3p & GTCCATGGTATTCAGTTCTCTA \\
\hline gga-miR-146b-5p & TGAGAACTGAATTCCATAGGCGTT \\
\hline gga-miR-30b-5p & TGTAAACATCCTACACTCAGCT \\
\hline gga-miR-9-5p & TCTTTGGTTATCTAGCTGTATGA \\
\hline gga-miR-1559-5p & TTCGATGCTTGTATGCTACTCC \\
\hline gga-miR-107-3p & AGCAGCATTGTACAGGGCTATCA \\
\hline gga-miR-455-5p & TGTGCCCTTGGACTACATCGTG \\
\hline gga-miR-30e-3p & CTTTCAGTCGGATGTTTACAGC \\
\hline gga-miR-140-5p & CAGTGGTTTTACCCTATGGTAG \\
\hline gga-miR-126-5p & CATTATTACTTTTGGTACGCG \\
\hline gga-miR-2188-5p & AAGGTCCAACCTCACATGTCCT \\
\hline gga-miR-106-3p & ACTGCAGTATAAGCACTTCTGGC \\
\hline gga-miR-130b-5p & СCTCTTTCCCTGTTGCACTACT \\
\hline gga-miR-144-3p & CTACAGTATAGATGATGTACTCT \\
\hline gga-miR-200a-5p & CATCTTACTAGACAGTGCTGGA \\
\hline gga-let-7b & TGAGGTAGTAGGTTGTGTGGTT \\
\hline gga-miR-125b-3p & ACAAGTCAGGCTCTTGGGACT \\
\hline gga-miR-1677-3p & TTGACTTCAGTAGGAGCAGGATT \\
\hline gga-miR-365-3p & TAATGCCCCTAAAAATCCTTAT \\
\hline gga-miR-146a-5p & TGAGAACTGAATTCCATGGGTTG \\
\hline gga-miR-19b-3p & TGTGCAAATCCATGCAAAACTG \\
\hline gga-miR-16-5p & TAGCAGCACGTAAATATTGGTG \\
\hline gga-miR-211 & TTCCCTTTGTCATCCTATGCCT \\
\hline gga-miR-460a-5p & CCTGCATTGTACACACTGTGTG \\
\hline gga-miR-17-5p & CAAAGTGCTTACAGTGCAGGTAG \\
\hline gga-miR-196-5p & TAGGTAGTTTCATGTTGTTGGG \\
\hline gga-miR-22-3p & AAGCTGCCAGTTGAAGAACTGT \\
\hline gga-miR-221-3p & AGCTACATTGTCTGCTGGGTTTC \\
\hline gga-miR-222a & AGCTACATCTGGCTACTGGGTCTCT \\
\hline gga-miR-7 & TGGAAGACTAGTGATTTTGTTGTT \\
\hline gga-miR-1a-3p & TGGAATGTAAAGAAGTATGTAT \\
\hline gga-miR-214 & ACAGCAGGCACAGACAGGCAGT \\
\hline gga-miR-454-3p & TAGTGCAATATTGCTTATAGGGTCT \\
\hline gga-miR-106-5p & AAAAGTGCTTACAGTGCAGGTAG \\
\hline gga-miR-140-3p & ACCACAGGGTAGAACCACGGAC \\
\hline gga-miR-429-3p & TAATACTGTCTGGTAATGCCGT \\
\hline gga-miR-181b-5p & AACATTCATTGCTGTCGGTGGGT \\
\hline gga-let-7c-5p & TGAGGTAGTAGGTTGTATGGTT \\
\hline gga-miR-205b & СCCTTCATTCCACCGGAATCTG \\
\hline gga-let-7k-5p & TGAGGTAGTAGATTGAATAGTT \\
\hline
\end{tabular}

\begin{tabular}{ll}
\hline Continuous the above Table 3 \\
\hline gga-miR-23b-3p & ATCACATTGCCAGGGATTTCCA \\
gga-miR-20b-5p & CAAAGTGCTCATAGTGCAGGTAG \\
gga-miR-7b & TGGAAGACTAGTGATTTTGTTGTT \\
gga-miR-181a-5p & AACATTCAACGCTGTCGGTGAGT \\
gga-miR-451 & AAACCGTTACCATTACTGAGTTT \\
gga-miR-30c-5p & TGTAAACATCCTACACTCTCAGCT \\
gga-miR-218-5p & TTGTGCTTGATCTAACCATGT \\
gga-miR-24-3p & TGGCTCAGTTCAGCAGGAACAG \\
gga-miR-20a-5p & TAAAGTGCTTATAGTGCAGGTAG \\
gga-miR-30e-5p & TGTAAACATCCTTGACTGGAAGCT \\
gga-miR-125b-5p & TCCCTGAGACCCTAACTTGTGA \\
gga-miR-130b-3p & CAGTGCAATAATGAAAGGGCGT \\
gga-miR-30a-5p & TGTAAACATCCTCGACTGGAAGCT \\
gga-miR-456-3p & CAGGCTGGTTAGATGGTTGTCT \\
gga-miR-103-3p & AGCAGCATTGTACAGGGCTATGA \\
gga-miR-128-3p & TCACAGTGAACCGGTCTCTTT \\
gga-miR-101-3p & GTACAGTACTGTGATAACTGAA \\
gga-miR-16c-5p & TAGCAGCACGTAAATACTGGAG \\
gga-miR-27b-3p & TTCACAGTGGCTAAGTTCTGC \\
gga-let-7j-5p & TGAGGTAGTAGGTTGTATAGTT \\
gga-let-7g-5p & TGAGGTAGTAGTTTGTACAGTT \\
gga-miR-126-3p & TCGTACCGTGAGTAATAATGCG \\
gga-miR-30d & TGTAAACATCCCCGACTGGAAGCT \\
gga-miR-200a-3p & TAACACTGTCTGGTAACGATGTT \\
gga-miR-200b-3p & TAATACTGCCTGGTAATGATGAT \\
gga-let-7i & TGAGGTAGTAGTTTGTGCTGTT \\
gga-miR-199-5p & CCCAGTGTTCAGACTACCTGTTC \\
gga-let-7f-5p & TGAGGTAGTAGATTGTATAGTT \\
gga-miR-92-3p & TATTGCACTTGTCCCGGCCTGT \\
gga-miR-26a-5p & TTCAAGTAATCCAGGATAGGCT \\
gga-miR-21-5p & TAGCTTATCAGACTGATGTTGAC \\
gga-miR-199-3p & ACAGTAGTCTGCACATTGGTT \\
gga-miR-10b-5p & TACCCTGTAGAACCGAATTTGT \\
gga-miR-99a-5p & AACCCGTAGATCCGATCTTGT \\
gga-miR-146c-5p & TGAGAACTGAATTCCATGGACTG \\
gga-miR-100-5p & AACCCGTAGATCCGAACTTGTG \\
gga-miR-10a-5p & TACCCTGTAGATCCGAATTTGT \\
gga-miR-205a & TCCTTCATTCCACCGGAGTCTG \\
gga-miR-148a-3p & TCAGTGCACTACAGAACTTTGT \\
gga-miR-203a & GTGAAATGTTTAGGACCACTTG \\
gga-miR-1682* & TGGGTCAGATGGAGCTGAGGGT \\
\hline & \\
\end{tabular}

* represent the KRC-specific miRNA.

\subsection{Differential Expression Profiles of Conserved Mirnas Between HXC and KRC}

To compare the differential expression of miRNAs in the follicle of $\mathrm{HXC}$ versus KRC chickens, the numbers of miRNAs in each group samples were normalized to the total number of reads. The expression of one KRC-specific miRNAs was not significant in KRC chickens compared to HXC counterparts. So only the differentially expressed miRNAs in follicle were showed in table 4. In total, 11 miRNAs were considered to be differentially expressed $(P<$ $0.05)$, with 5 up-regulated and 6 down-regulated. Nine miRNAs had more than two fold expression changes $(\mid \log 2$ (fold-change) $\mid>=1.0$ ) from KRC to HXC (Table 4). We also confirmed the expression patterns by RT-qPCR which of four differentially expressed miRNAs in follicle (Figure 2), the results of which correlated to the RPKM values estimated by RNA sequencing $(\mathrm{r}=0.81)$. 
Table 4. The differentially expressed miRNAs between HXC and KRC.

\begin{tabular}{lllll}
\hline miR_name & miR_seq & Fold Change (KRC/HXC) & log2(Fold Change) & P-value \\
\hline gga-miR-1623 & ACCGCAGGCACAGACAGGCAGT & 9.09 & 3.18 & 0.00000 \\
gga-miR-6544-3p & AGTTGTATTTCTTTTCTGACAG & 3.30 & 1.72 & 0.00871 \\
gga-miR-1458 & TTCCTGTGATGCTCATGAGA & 3.04 & 1.60 & 0.00281 \\
gga-miR-1559-3p & AGTTACATGTATGCATCGAGCA & 2.81 & 1.49 & 0.00536 \\
gga-miR-199b & CAGTAGTCTGCACATT & 2.34 & 1.23 & 0.00628 \\
gga-miR-144-5p & GGATATCATCATATACTGTAAGT & 0.59 & -0.77 & 0.04962 \\
gga-miR-6599-3p & TGACGGATCCTGGCTCCCTCCG & 0.51 & -0.98 & 0.03224 \\
gga-miR-1731-5p & ACTTGACTGCAGGCACTGCTGCT & 0.50 & -1.01 & 0.03513 \\
gga-miR-184-3p & TGGACGGAGAACTGATAAGGGT & 0.36 & -1.46 & 0.03880 \\
gga-miR-1798-5p & AACGTGACACTTTAGAAAACCT & 0.26 & -1.95 & 0.03156 \\
gga-miR-1798-3p & TTTTCAGAAGTGTAGCGTTTA & 0.12 & -3.11 & 0.00003 \\
\hline
\end{tabular}

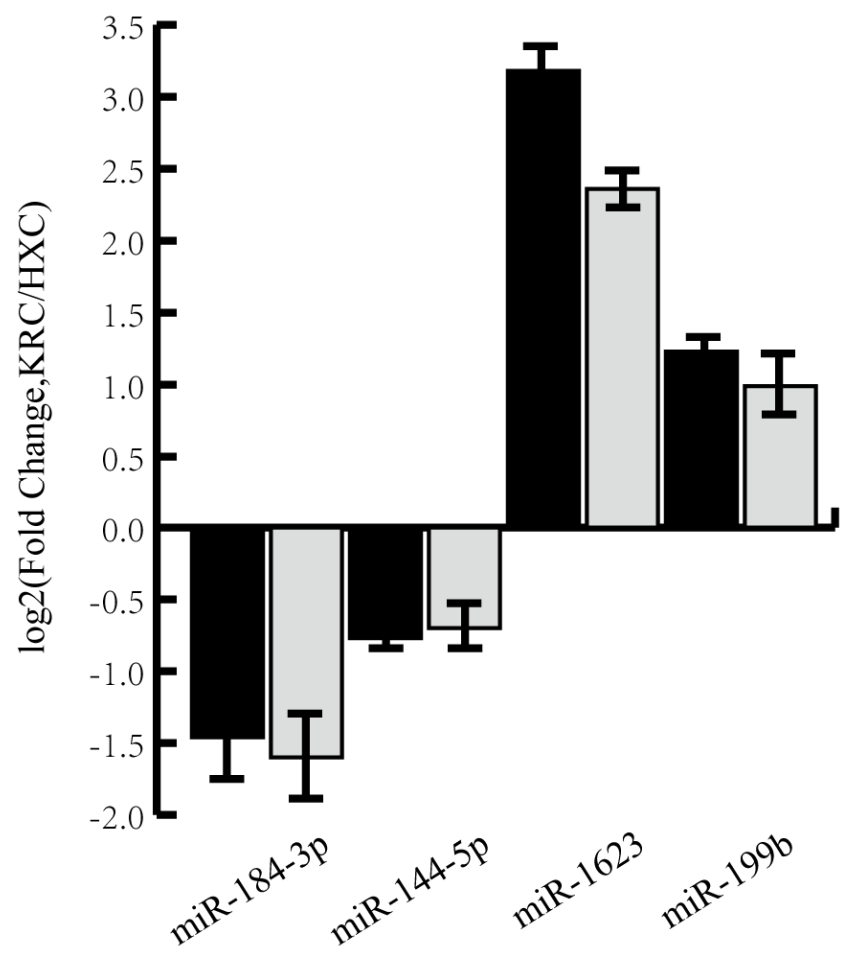

Figure 2. The expression patterns by RT-qPCR compared with RNA-seq of differentially expressed miRNAs.

\subsection{Target Prediction and Functional Analysis of Differential Expression Mirnas}

To further explore the roles of differentially expressed miRNAs, putative target genes of the most differentially expressed 9 miRNAs $(\mid \log 2$ (fold-change) $\mid>=1.0$ ) were predicted by integrating TargetScan and miRanda. In total, 8250 common target genes were found (data is not shown) which include FZD4, WNT4, BMP and EGF of which related hair follicle development (Table 5).

Table 5. The differentially expressed miRNAs target genes annotation.

\begin{tabular}{llll}
\hline Gene Name & Ensembl Gene ID & Involved KEGG pathways & Associated miRNAs \\
\hline FZD4 & ENSGALG00000017242 & Wnt signaling pathway & gga-miR-1623, gga-miR-184-3p, gga-miR-1458 \\
$W N T 4$ & ENSGALG00000004790 & mTOR signaling pathway & gga-miR-1623 \\
$E G F$ & ENSGALG00000012155 & MAPK signaling pathway & gga-miR-184-3p, gga-miR-199b \\
$B M P 2 / 4$ & ENSGALG00000012429 & TGF-beta signaling pathway & miR-184-3p, miR-1559-3p \\
\hline
\end{tabular}

GO annotation showed the putative target genes were significantly enriched (counts $>30, P<0.05$ ) in biological processes (BP) (Table 6), Cellular component (CC) (Table 7) and Molecular function (MF) (Table 8). The KEGG analysis
(Table 9) suggested that Focal adhesion, Phosphatidylinositol signaling system, ECM-receptor interaction, Inositol phosphate metabolism, Oocyte meiosis and Ubiquitin mediated proteolysis were the most enriched 
pathways (counts $>50, P<0.01)$.

Table 6. GO analysis of the putative target genes in biological processes.

\begin{tabular}{|c|c|c|}
\hline GO term in biological processes & gene count & P-value \\
\hline cell migration & 78 & 0.00003 \\
\hline protein autophosphorylation & 76 & 0.00006 \\
\hline signal transduction & 166 & 0.00006 \\
\hline intracellular protein transport & 106 & 0.00013 \\
\hline peptidyl-tyrosine phosphorylation & 33 & 0.00017 \\
\hline peptidyl-serine phosphorylation & 70 & 0.00028 \\
\hline activation of GTPase activity & 41 & 0.00035 \\
\hline cell-matrix adhesion & 36 & 0.00066 \\
\hline positive regulation of protein kinase B signaling & 37 & 0.00300 \\
\hline positive regulation of JNK cascade & 30 & 0.00450 \\
\hline $\begin{array}{l}\text { positive regulation of I-kappaB kinase/NF-kappaB } \\
\text { signaling }\end{array}$ & 68 & 0.00460 \\
\hline cell adhesion & 97 & 0.00500 \\
\hline axon guidance & 54 & 0.00740 \\
\hline extracellular matrix organization & 49 & 0.00760 \\
\hline cilium assembly & 62 & 0.00980 \\
\hline $\begin{array}{l}\text { positive regulation of NF-kappaB transcription } \\
\text { factor activity }\end{array}$ & 43 & 0.00990 \\
\hline single organismal cell-cell adhesion & 30 & 0.01000 \\
\hline $\begin{array}{l}\text { positive regulation of canonical Wnt signaling } \\
\text { pathway }\end{array}$ & 35 & 0.01100 \\
\hline $\begin{array}{l}\text { positive regulation of transcription from RNA } \\
\text { polymerase II promoter }\end{array}$ & 273 & 0.01200 \\
\hline positive regulation of protein binding & 32 & 0.01200 \\
\hline positive regulation of cell migration & 74 & 0.01300 \\
\hline $\begin{array}{l}\text { transmembrane receptor protein tyrosine kinase } \\
\text { signaling pathway }\end{array}$ & 46 & 0.01500 \\
\hline intracellular signal transduction & 148 & 0.01600 \\
\hline $\begin{array}{l}\text { positive regulation of peptidyl-serine } \\
\text { phosphorylation }\end{array}$ & 36 & 0.01600 \\
\hline integrin-mediated signaling pathway & 36 & 0.01600 \\
\hline $\begin{array}{l}\text { positive regulation of proteasomal } \\
\text { ubiquitin-dependent protein catabolic process }\end{array}$ & 33 & 0.01900 \\
\hline xenophagy & 40 & 0.02000 \\
\hline $\begin{array}{l}\text { positive regulation of phosphatidylinositol 3-kinase } \\
\text { signaling }\end{array}$ & 35 & 0.02100 \\
\hline $\begin{array}{l}\text { positive regulation of neuron projection } \\
\text { development }\end{array}$ & 30 & 0.02100 \\
\hline post-embryonic development & 37 & 0.02300 \\
\hline positive regulation of transcription, DNA-templated & 134 & 0.02600 \\
\hline innate immune response & 75 & 0.03200 \\
\hline $\begin{array}{l}\text { protein ubiquitination involved in } \\
\text { ubiquitin-dependent protein catabolic process }\end{array}$ & 68 & 0.03600 \\
\hline protein polyubiquitination & 44 & 0.03700 \\
\hline cellular response to lipopolysaccharide & 30 & 0.03900 \\
\hline positive regulation of cell proliferation & 110 & 0.04200 \\
\hline regulation of Rho protein signal transduction & 34 & 0.04500 \\
\hline endocytosis & 45 & 0.04700 \\
\hline protein phosphorylation & 60 & 0.04700 \\
\hline $\begin{array}{l}\text { negative regulation of transcription, } \\
\text { DNA-templated }\end{array}$ & 111 & 0.04700 \\
\hline
\end{tabular}

Table 7. GO analysis of the putative target genes in Cellular component.

\begin{tabular}{lll}
\hline GO term in Cellular component & gene count & P-value \\
\hline nucleoplasm & 685 & 0.00000 \\
cytoplasm & 1337 & 0.00000 \\
centrosome & 183 & 0.00000 \\
plasma membrane & 726 & 0.00001 \\
PML body & 47 & 0.00002 \\
cytosol & 494 & 0.00013 \\
early endosome & 82 & 0.00048 \\
proteinaceous extracellular matrix & 100 & 0.00052 \\
extracellular exosome & 959 & 0.00054 \\
\hline
\end{tabular}

\begin{tabular}{lll}
\hline GO term in Cellular component & gene count & P-value \\
\hline perinuclear region of cytoplasm & 175 & 0.00069 \\
cell junction & 100 & 0.00082 \\
cell-cell junction & 63 & 0.00150 \\
spindle pole & 34 & 0.00210 \\
centriole & 42 & 0.00210 \\
receptor complex & 59 & 0.00230 \\
cell surface & 169 & 0.00240 \\
extrinsic component of membrane & 40 & 0.00380 \\
trans-Golgi network & 66 & 0.00620 \\
basement membrane & 35 & 0.00750 \\
postsynaptic membrane & 54 & 0.00120 \\
ciliary basal body & 38 & 0.01200 \\
Golgi apparatus & 237 & 0.01400 \\
endomembrane system & 44 & 0.01500 \\
cytoplasmic vesicle & 51 & 0.02300 \\
apical plasma membrane & 76 & 0.03100 \\
membrane & 417 & 0.03600 \\
recycling endosome & 40 & 0.03700 \\
lamellipodium & 60 & 0.04300 \\
blood microparticle & 35 & 0.04300 \\
\hline
\end{tabular}

Table 8. GO analysis of the putative target genes in Molecular function.

\begin{tabular}{lll}
\hline GO term in Molecular function & gene count & P-value \\
\hline ATP binding & 679 & 0.00000 \\
metal ion binding & 416 & 0.00000 \\
zinc ion binding & 471 & 0.00002 \\
phosphatidylinositol binding & 50 & 0.00031 \\
protein serine/threonine kinase activity & 122 & 0.00044 \\
ubiquitin-protein transferase activity & 90 & 0.00065 \\
protein kinase activity & 67 & 0.00069 \\
non-membrane spanning protein tyrosine kinase & 30 & 0.00250 \\
activity & 100 & 0.00280 \\
GTPase activator activity & 57 & 0.00460 \\
transcription regulatory region DNA binding & 47 & 0.00580 \\
receptor activity & 39 & 0.00760 \\
ligase activity & 39 & 0.00760 \\
thiol-dependent ubiquitin-specific protease activity & 62 & 0.01000 \\
ATPase activity & 35 & 0.01200 \\
receptor signaling protein serine/threonine kinase & 35 & \\
activity & 30 & 0.01300 \\
extracellular matrix structural constituent & 37 & 0.02200 \\
receptor binding & 30 & 0.02400 \\
Rab GTPase binding & 145 & 0.02700 \\
chromatin binding & 31 & 0.03300 \\
microtubule motor activity & 63 & 0.03900 \\
\hline signal transducer activity &
\end{tabular}

Table 9. KEGG analysis of the putative target genes.

\begin{tabular}{lcc}
\hline KEGG Term & Count & P-value \\
\hline Focal adhesion & 137 & 0.00001 \\
Phosphatidylinositol signaling system & 73 & 0.00003 \\
ECM-receptor interaction & 59 & 0.00015 \\
Inositol phosphate metabolism & 55 & 0.00026 \\
Oocyte meiosis & 67 & 0.00110 \\
Ubiquitin mediated proteolysis & 87 & 0.00690 \\
Cell cycle & 77 & 0.01700 \\
Endocytosis & 153 & 0.03700 \\
p53 signaling pathway & 44 & 0.04100 \\
\hline
\end{tabular}

\section{Discussion}

In this study, we detected 11 differential expressed miRNAs that were enriched in the KRC and $\mathrm{HXC}$ libraries and obtained several predicted target genes that may play different roles in 
hair follicles formation and development. We also identified several pathways associated with hair follicle cell development, including Focal adhesion, Phosphatidylinositol signaling system, ECM-receptor interaction.

Our analysis of the most abundant mature miRNAs with raw reads $>=10$ in the KRCs and HXCs identified 290 miRNAs that were common in the two groups. Our analysis of the differential expressed miRNAs revealed 11 miRNAs (table 2) in two groups. These differential expressed miRNAs including miR-1623, miR-184-3p, miR-199b. The miR-184 inhibition argonaute 2 protein expression [22] and the growth of hair follicles [23]. And miRNA-199b has an important role in skin and hair follicle development $[24,25]$. The miR-1623 target genes that played important roles in $\mathrm{Wnt} / \beta$-catenin pathway [19]. Through qRT-PCR, we confirmed miR-1623 target gene the WNT4 in chicken and WNT4 showed the higher expression in HXCs (Figure 3). Thus, the miR-1623 has an effect on WNT4 and involved in hair follicle cell development. We also identified several target genes, such as those encoding $F Z D 4$ and $E G F$, which are related to hair follicle development in chicken. These miRNAs target genes involved several signaling pathways, including WNT, TGF- $\beta$, EGF, FGF, BMP, Hox signaling pathway [26-30]. These signaling pathways regulated and transformed hair follicle development in different stages. The WNT and BMP signaling pathways related to differentiation of keratin cell and regulation of the hair shaft formation [31]. And the TGF- $\beta$ pathway control growth of hair follicles [27].

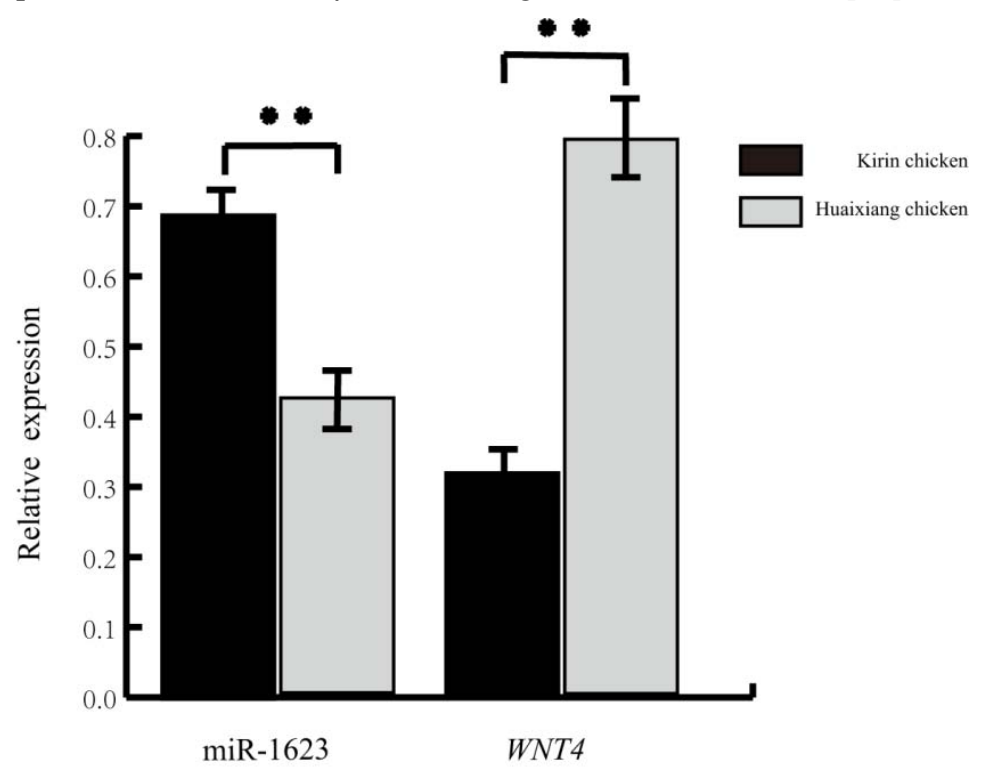

Figure 3. The miR-1623 target gene expression.

Many complex factors support hair follicle cell development. To further investigate the functions of miRNAs and their target genes more experiments need to be performed and miRNA knockdown to identify target genes expression levels.

\section{Conclusion}

In conclusion, we identified several miRNAs such as miR-1623, miR-184-3p, miR-199b, and their target genes WNT4, FZD4 and EGF which may be involved in hair follicle development in chicken. These data provide a strong foundation for the study of hair follicle development in chicken at the molecular levels.

\section{Acknowledgements}

This work was supported by the Guangdong Ocean University Research System (101402/R17030) and Program of Guangdong key laboratory of genomics and molecular breeding of agricultural animals. We have no competing interests in this research.

\section{References}

[1] Bartel DP: MicroRNAs: target recognition and regulatory functions. Cell 2009, 136(2):215-233.

[2] Ambros V: The function of animal MicroRNAs. Nature 2004, 431(7006):350-355.

[3] Squadrito ML, Baer C, Burdet F, Maderna C, Gilfillan GD, Lyle R, Ibberson M, De Palma M: Endogenous RNAs Modulate MicroRNA Sorting to Exosomes and Transfer to Acceptor Cells. Cell Reports 2014, 8(5):1432-1446.

[4] Villarroyabeltri C, Gutierrezvazquez C, Sanchezcabo F, Perezhernandez D, Vazquez J, Martincofreces NB, Martinezherrera DJ, Pascualmontano A, Mittelbrunn M, Sanchezmadrid F: Sumoylated hnRNPA2B1 controls the sorting of miRNAs into exosomes through binding to specific motifs. Nature Communications 2013, 4:2980-2980.

[5] Hochfeld LM, Anhalt T, Hofmann A, Fricker N, Nothen MM, Heilmannheimbach S: 192 MicroRNAs and their regulatory interactions in the human hair follicle. Journal of Investigative Dermatology 2016, 136(9). 
[6] Li C, Ren L, Wang Y, Zhong J, Huang L, Lin Y, Zi X, Zheng Y: 0345 Construction and functional analysis of expression vector and miRNA interference vectors of Gsdma of Tibetan sheep. Journal of Animal Science 2016, 94:165-166.

[7] Wade B, Cummins ML, Keyburn AL, Crowley TM: Isolation and detection of microRNA from the egg of chickens. BMC Research Notes 2016, 9(1):283.

[8] Mai M, Jin L, Tian S, Liu R, Huang W, Tang Q, Ma J, Jiang A, Wang X, Hu Y: Deciphering the microRNA transcriptome of skeletal muscle during porcine development. PeerJ 2016, 4.

[9] Wu N, Gaur U, Zhu Q, Chen B, Xu Z, Zhao X, Yang M, Li D: Expressed microRNA associated with high rate of egg production in chicken ovarian follicles. Animal Genetics 2017, 48(2):205-216

[10] Feng C, Gao Y, Dorshorst B, Song C, Gu X, Li Q, Li J, Liu T, Rubin C, Zhao Y: A cis-regulatory mutation of PDSS2 causes silky-feather in chickens. PLOS Genetics 2014, 10(8).

[11] Cooke TF, Fischer CR, Wu P, Jiang TX, Xie KT, Kuo J, Doctorov E, Zehnder A, Khosla C, Chuong CM: Genetic Mapping and Biochemical Basis of Yellow Feather Pigmentation in Budgerigars. Cell 2017, 171(2):427-439.e421.

[12] Li A, Figueroa S, Jiang TX, Wu P, Widelitz R, Nie Q, Chuong $\mathrm{CM}$ : Diverse feather shape evolution enabled by coupling anisotropic signalling modules with self-organizing branching programme. Nature Communications 2017, 8: ncomms14139.

[13] Lowe CB, Clarke JA, Baker AJ, Haussler D, Edwards SV: Feather development genes and associated regulatory innovation predate the origin of Dinosauria. Molecular Biology and Evolution 2015, 32(1):23-28.

[14] Ng CS, Wu P, Foley J, Foley A, Mcdonald MN, Juan W, Huang C, Lai Y, Lo W, Chen C: The Chicken Frizzle Feather Is Due to an $\alpha$-Keratin (KRT75) Mutation That Causes a Defective Rachis. PLOS Genetics 2012, 8(7).

[15] Wu P, Ng CS, Yan J, Lai Y, Chen C, Lai Y, Wu S, Chen J, Luo W, Widelitz RB: Topographical mapping of $\alpha$ - and $\beta$-keratins on developing chicken skin integuments: Functional interaction and evolutionary perspectives. Proceedings of the National Academy of Sciences of the United States of America 2015, 112(49).

[16] Alibardi L: Review: cornification, morphogenesis and evolution of feathers. Protoplasma 2017, 254(3):1259-1281.

[17] Yi R, Ocarroll D, Pasolli HA, Zhang Z, Dietrich FS, Tarakhovsky A, Fuchs E: Morphogenesis in skin is governed by discrete sets of differentially expressed microRNAs. Nature Genetics 2006, 38(3):356-362.

[18] Mardaryev AN, Ahmed MI, Vlahov N, Fessing MY, Gill JH, Sharov AA, Botchkareva NV: Micro-RNA-31 controls hair cycle-associated changes in gene expression programs of the skin and hair follicle. The FASEB Journal 2010, 24(10):3869-3881.
[19] Zhang L, Nie Q, Su Y, Xie X, Luo W, Jia X, Zhang X: MicroRNA profile analysis on duck feather follicle and skin with high-throughput sequencing technology. Gene 2013, 519(1):77-81.

[20] Mestdagh P, Van VP, De WA, Muth D, Westermann F, Speleman F, Vandesompele J: A novel and universal method for microRNA RT-qPCR data normalization. Genome Biology 2009, 10(6): R64.

[21] Livak KJ, Schmittgen TD: Analysis of relative gene expression data using real-time quantitative PCR and the 2(-Delta Delta C(T)) Method. Methods 2001, 25(4):402-408.

[22] Roberts JC, Warren RB, Griffiths CEM, Ross K: Expression of microRNA-184 in keratinocytes represses argonaute 2. Journal of Cellular Physiology 2013, 228(12):2314-2323.

[23] Yu J, Ryan DG, Getsios S, Oliveirafernandes M, Fatima A, Lavker RM: MicroRNA-184 antagonizes microRNA-205 to maintain SHIP2 levels in epithelia. Proceedings of the National Academy of Sciences of the United States of America 2008, 105(49):19300-19305.

[24] Liu Z, Xiao H, Li H, Zhao Y, Lai S, Yu X, Cai T, Du C, Zhang W, Li J: Identification of Conserved and Novel microRNAs in Cashmere Goat Skin by Deep Sequencing. PLOS ONE 2012, 7(12).

[25] Andl T, Murchison EP, Liu F, Zhang Y, Yuntagonzalez M, Tobias JW, Andl CD, Seykora JT, Hannon GJ, Millar SE: The miRNA-Processing Enzyme Dicer Is Essential for the Morphogenesis and Maintenance of Hair Follicles. Current Biology 2006, 16(10):1041-1049.

[26] Fu J, Hsu W: Epidermal Wnt controls hair follicle induction by orchestrating dynamic signaling crosstalk between the epidermis and dermis. Journal of Investigative Dermatology 2013, 133(4):890-898.

[27] Wollina U, Lange D, Funa K, Paus R: Expression of transforming growth factor beta isoforms and their receptors during hair growth phases in mice. Histology and Histopathology 1996, 11(2):431.

[28] Murillas R, Larcher F, Conti CJ, Santos M, Ullrich A, Jorcano JL: Expression of a dominant negative mutant of epidermal growth factor receptor in the epidermis of transgenic mice elicits striking alterations in hair follicle development and skin structure. The EMBO Journal 1995, 14(21):5216-5223.

[29] Suzuki S, Ota Y, Ozawa K, Imamura T: Dual-Mode Regulation of Hair Growth Cycle by Two Fgf-5 Gene Products. Journal of Investigative Dermatology 2000, 114(3):456-463.

[30] Jianghong WU, Zhang Y, Zhang J, Chang Z, Jinquan LI, Yan Z, Husile, Zhang W: Hoxc13//-catenin Correlation with Hair Follicle Activity in Cashmere Goat. Journal of Integrative Agriculture 2012, 11(7):1159-1166.

[31] Schmidtullrich R, Paus R: Molecular principles of hair follicle induction and morphogenesis. Bio Essays 2005, 27(3):247-261. 